\title{
What Is the Optimum Window Width when Calculating the Interaction between RR Intervals and Systolic Pressure Levels?
}

\author{
EJ Bowers, A Murray \\ Regional Medical Physics Department, Freeman Hospital, Newcastle upon Tyne, UK
}

\begin{abstract}
The ability to reliably measure phase between changes in heart rate and blood pressure is important when developing cardiovascular models.

This study recorded 20 subjects breathing normally for periods of five minutes. RR intervals and systolic pressure $(S P)$ levels were extracted from ECG and blood pressure signals. Phase was calculated using cross spectral analysis using four different windowing techniques.

An analysis of variance showed that phase was not affected by windowing techniques. However, variance of phase reduced as window width reduced and the number of windows increased. We concluded that 5 Hanning windows of width $100 \mathrm{~s}$ (with 50\% overlap) produce a good compromise between minimum variance and maximum frequency resolution.
\end{abstract}

\section{Introduction}

RR interval and systolic pressure (SP) levels are mainly kept in balance by the baroreflex feedback loop. The integrity of the loop is traditionally measured by the ratio of changes in RR intervals to changes in SP levels, that is its gain, also known as baroreflex sensitivity (BRS). BRS has been shown to be clinically useful [1].

Another aspect of the interaction between RR intervals and SP levels is the phase delay. In the past there has been lack of clarity in the literature reporting phase as to which points it is measured between [2]. Consequently there has been little investigation into the clinical usefulness of phase.

The two main techniques used for calculating phase delay are cross correlation in the time domain and cross spectral analysis in the frequency domain. The latter is a more generic version of the former.

Due to RR interval and SP level data being nonstationary, when calculating the cross spectrum, windowing techniques with different shapes, widths or overlaps have to be used. The shape of a window affects its frequency spectrum. The multiplication of the windowing function with the signal in the time domain is equivalent to the convolution of a window's frequency spectrum with the signal in the frequency domain. The window shape may then introduce small erroneous frequency components to the signal which may contain incorrect phase information. The wider the window, the better the frequency resolution, but the less statistically reliable the phase data, since the mean phase must be calculated from a smaller number of windows. Overlapping increases the number of windows from which mean phase can be calculated, allowing centring of features which might otherwise appear only at the edge giving statistically more reliable results.

This study aimed to: 1) compare rectangular and Hanning (raised cosine) windows; 2) investigate the optimum window width for reconciling the conflicting effects of resolution and statistical reliability.

\section{Methods}

\subsection{Subjects}

Twenty subjects (10 females, 10 males) aged $35 \pm 10$ years were recruited. Subjects considered themselves free of cardiovascular or respiratory problems. All subjects gave written informed consent before taking part.

\subsection{Study protocol}

Subjects made themselves comfortable semi-supine and were asked to breathe normally for 5 minutes (300 s).

\subsection{Physiological data}

Throughout the study, subjects had their ECG recorded from a single lead ECG, and their non-invasive blood pressure by a Finapres device [3]. All physiological data were sampled at $500 \mathrm{~Hz}$ and recorded to computer.

\subsection{Data processing}

RR intervals and SP levels were automatically extracted from the ECG and Finapres signals respectively, and visually checked. Interpolation was applied when the Finapres device performed a short 'servo self-adjustment', usually for 2 beats out of 70. The Berger algorithm [4] was applied to regularly sample the data at $16 \mathrm{~Hz}$. The data length of complete RR intervals 
was always just short of $300 \mathrm{~s}$.

Prior to frequency analysis, RR interval and SP level data were split into $150 \mathrm{~s}, 100 \mathrm{~s}, 75 \mathrm{~s}, 60 \mathrm{~s}$, and $50 \mathrm{~s}$ sections which were either non overlapping or $50 \%$ overlapping. This resulted in 2, 3, 4, 5, or 6 non overlapping sections (figure 1) or 3, 5, 7, 9 or $1150 \%$ overlapping sections. The mean was removed from the data in each section and a number of zeroes added to the last section to bring the total data length back to $300 \mathrm{~s}$. Lastly each section was multiplied by a rectangular or Hanning window of the correct width.

The Fourier transform was performed on each windowed section of data to give $\operatorname{RR}(\omega)$ and $\operatorname{SP}(\omega)$, from which was calculated the:

1) Power spectrum of RR intervals $R R(\omega)^{2}$.

2) Power spectrum of SP levels $\operatorname{SP}(\omega)^{2}$.
3) Cross spectrum between RR intervals and SP levels $\operatorname{RRSP}(\omega)=\operatorname{RR} *(\omega) \times \operatorname{SP}(\omega) .\left(^{*}=\right.$ complex conjugate $)$

The average of these spectra was calculated for the whole dataset, resulting in frequency resolutions of $0.007 \mathrm{~Hz}, 0.01 \mathrm{~Hz}, 0.013 \mathrm{~Hz}, 0.017 \mathrm{~Hz}$ and $0.02 \mathrm{~Hz}$ for window widths of $150 \mathrm{~s}, 100 \mathrm{~s}, 75 \mathrm{~s}, 60 \mathrm{~s}$, and $50 \mathrm{~s}$.

Direct comparison between frequency components across different window widths is difficult as frequency resolutions differ. Figure 2 shows how these comparisons were made.

To confirm the statistical reliability of the average cross spectral data, coherence was calculated $|\operatorname{RRSP}(\omega)|^{2} /\left(\operatorname{RR}(\omega)^{2} \mathrm{xSP}(\omega)^{2}\right)$ at each frequency.

Analysis was conducted at frequencies where coherence was greater than 0.5 for all windows (figure 3 ). At these frequencies the phase and variance of this phase were examined for each window width.
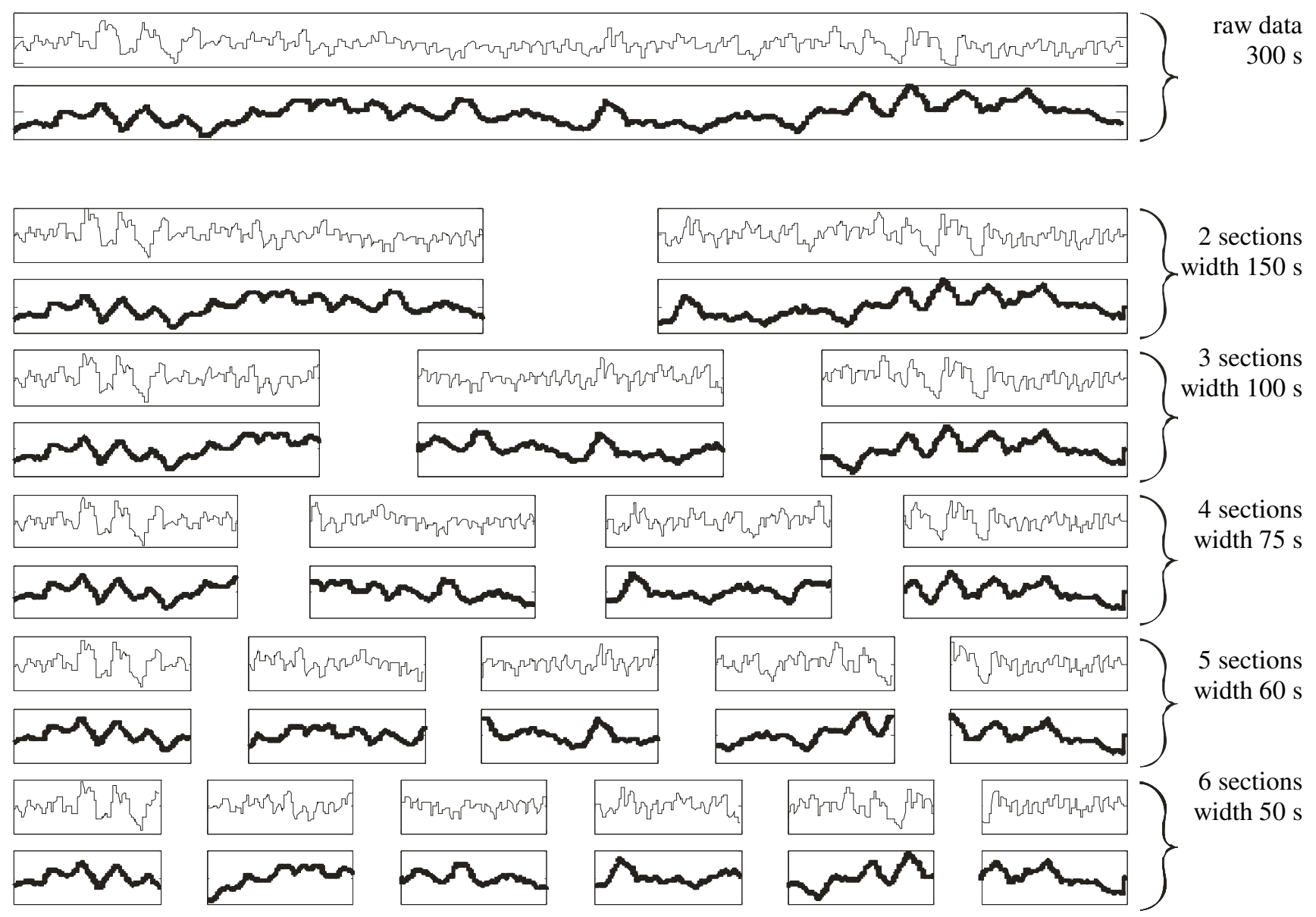

Figure 1. Data from one subject: narrow lines represent RR intervals and bold lines represent SP levels. The raw RR interval data is between 0.9 and $1.1 \mathrm{~s}$ and the raw SP level data is between 100 and $140 \mathrm{mmHg}$. Each section of data has had the mean of that data removed, the RR interval data is between -0.1 and $0.1 \mathrm{~s}$ and the SP level is between -20 and $20 \mathrm{mmHg}$. Zeros have been added to the final portion of data so the final section is the same length as the other sections. The next stage in the analysis process is to multiply each section of data by either a rectangular or Hanning window. 


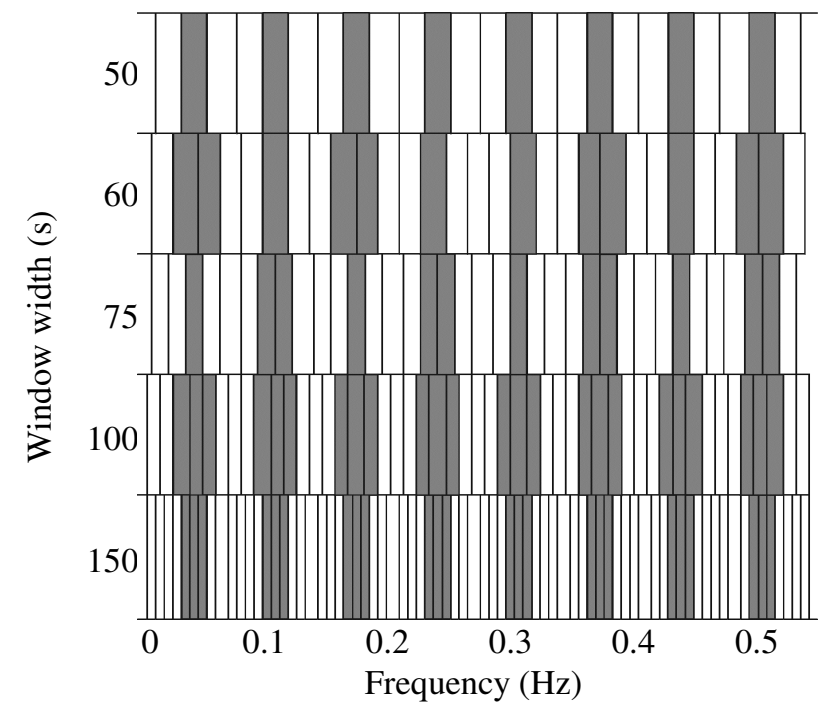

Figure 2. The frequency components that are compared when calculating phase. These same frequency components are shown in figure 3

\section{Results}

\subsection{Coherence}

The percentage of frequency components with a coherence greater than 0.5 across all windowing techniques is summarized in table 1 .

The percentage of frequencies from the $50 \mathrm{~s}$ window with a coherence greater than 0.5 across all windows for all subjects, was $14 \%, 24 \%, 19 \%$ and $25 \%$ respectively, for non overlapping rectangular, non overlapping Hanning, 50\% overlapping rectangular and 50\% overlapping Hanning windows.

\subsection{Different windows}

An analysis of variance (ANOVA) showed there was no significant difference in phase between rectangular and Hanning windows taking into account subject and frequency. The $\mathrm{p}$ values were $0.8,0.7,0.6,0.2$ and 0.7 for windows of width $150 \mathrm{~s}, 100 \mathrm{~s}, 75 \mathrm{~s}, 60 \mathrm{~s}$ and $50 \mathrm{~s}$ respectively.

\subsection{Variance of phase}

An analysis of the variances performed on a total of 694, 935, 746 and 987 frequency components for non overlapping rectangular and Hanning windows, and 50\% overlapping rectangular and Hanning windows showed that there was no significant difference in the phase measured between window widths but that variances were smaller for narrower windows (figure 4).

\section{Discussion and conclusions}

As would be expected a reduction in window width and hence more windows results in a reduction in variance for all four windowing techniques. When applying rectangular non overlapping windows the standard deviation began to plateau at width $75 \mathrm{~s}$ (equivalent to 4 windows). When using Hanning non overlapping windows it began to plateau at width $100 \mathrm{~s}$ (equivalent to 3 windows). In both cases it plateaued at $\sim 0.15$ rad. When applying rectangular $50 \%$ overlapping windows standard deviation plateaus at width $100 \mathrm{~s}$ (equivalent to 5 windows).(The standard deviation at this point was very similar to that calculated using the non overlapping techniques). When using Hanning 50\% overlapping windows the standard deviation began to plateau at width $100 \mathrm{~s}$ (equivalent to 5 windows) at $\sim 0.08$ rad, a considerably better result than those of the other techniques.

We were concerned that we artificially increased the variance by using more frequency components than may have been necessary. Therefore we repeated our calculations but reduced the three frequency components from the $100 \mathrm{~s}$ window to one. Interestingly these results were very similar to our original ones.

\begin{tabular}{lllll}
\hline $\begin{array}{l}\text { Window } \\
\text { Width (s) }\end{array}$ & $\begin{array}{l}\text { Non overlapping } \\
\text { rectangular window }\end{array}$ & $\begin{array}{l}\text { Non overlapping } \\
\text { Hanning window }\end{array}$ & $\begin{array}{l}50 \% \text { Overlapping } \\
\text { rectangular window }\end{array}$ & $\begin{array}{l}50 \% \text { Overlapping } \\
\text { Hanning window }\end{array}$ \\
\hline 150 & 69 & 73 & 60 & 59 \\
100 & 56 & 59 & 47 & 49 \\
75 & 47 & 52 & 41 & 42 \\
60 & 40 & 50 & 35 & 38 \\
50 & 37 & 45 & 35 & 36 \\
\hline
\end{tabular}

Table 1 . The percentage of frequency components that had a coherence greater than 0.5 


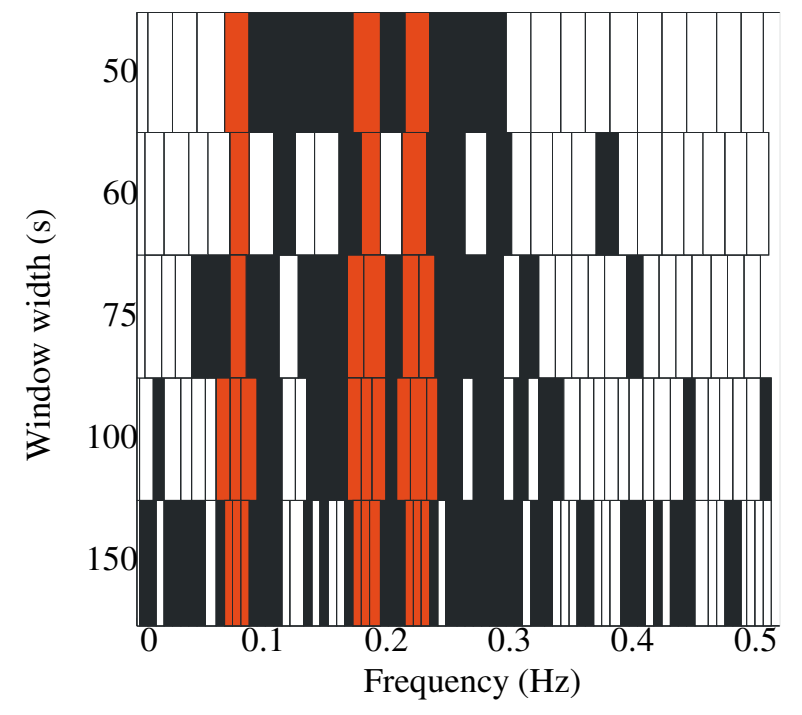

Figure 3. Coherence is greater than 0.5 where frequency components are coloured black or grey. Grey indicates components where coherence is greater than 0.5 for all windows. Where multiple frequency components are compared, all that were close to the reference component as explained in figure 2 had to have a coherence greater than 0.5 to be marked grey.

a)

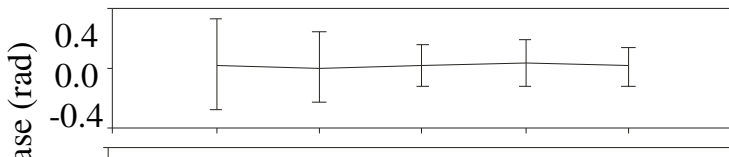

b)

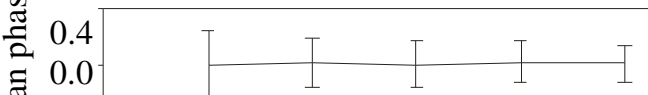

$-0.4$

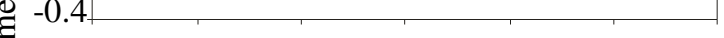

c)

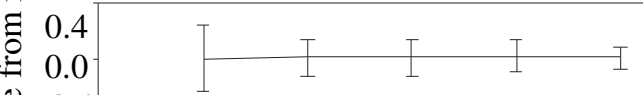

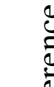

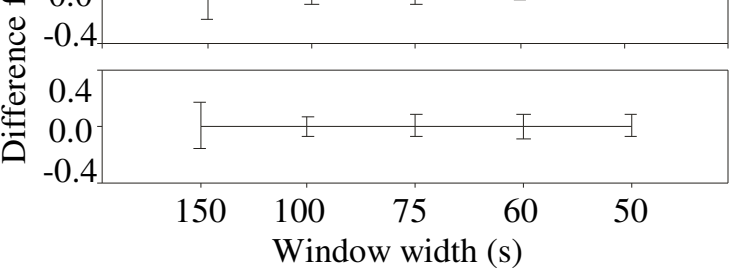

Figure 4. The mean \pm standard deviation from the mean phase. (a) Non overlapping rectangular window (b) Non overlapping Hanning window (c) $50 \%$ overlapping rectangular window (d) $50 \%$ overlapping Hanning window.
We conclude that there was no significant difference in phase calculated using either rectangular or Hanning windows. The 50\% overlapping Hanning window resulted in less variability of phase than all the other techniques. We conclude that when using the $50 \%$ overlapping Hanning window technique its optimum width was $100 \mathrm{~s}$. This window width resulted in a resolution of $0.01 \mathrm{~Hz}$ and a standard deviation of 0.07 rad.

\section{Acknowledgements}

The authors acknowledge the Institute of Physics and Engineering in Medicine (IPEM) who awarded Emma Bowers a PhD fellowship.

\section{References}

[1] La Rovere M, Bigger Jr JT, Marcus FI, Mortara A, Schwartz PJ. Baroreflex sensitivity and heart-rate variability in prediction of total cardiac mortality after myocardial infarction. The Lancet 1998;351:478-484.

[2] Bowers EJ, Murray A. Interaction between cardiac beat to beat interval changes and blood pressure changes. Clinical Autonomic Research 2004;14:92-98.

[3] Penaz J. Photoelectric measurement of blood pressure, volume and flow in the finger. In: Albert R, Vogt W, Helbig W, editors. 10th International Conference on Medical and Biological Engineering, Dresden, 1973:104.

[4] Berger RD, Akselrod S, Gordon D, Cohen RJ. An efficient algorithm for spectral analysis of heart rate variability. IEEE Transactions on Biomedical Engineering 1986;33:900-904.

Address for correspondence

Emma J. Bowers

Regional Medical Physics Department

Freeman Hospital

Newcastle upon Tyne NE7 7DN

UK

E-mail address: emma.bowers@ nuth.northy.nhs.uk 